\title{
Transgenic Targeting of Recombinant Rabies Virus Reveals Monosynaptic Connectivity of Specific Neurons
}

\author{
Aldis P. Weible, ${ }^{1 *}$ Leslie Schwarcz, ${ }^{2 *}$ Ian R. Wickersham, ${ }^{3}$ Leah DeBlander, ${ }^{1}$ Haiyan Wu, ${ }^{1}$ Edward M. Callaway, ${ }^{4}$ \\ H. Sebastian Seung, ${ }^{3}$ and Clifford G. Kentros ${ }^{1}$ \\ ${ }^{1}$ Department of Psychology, Institute of Neuroscience, University of Oregon, Eugene, Oregon 97403, 2 School of Medical Sciences, University of Auckland, \\ Auckland 1142, New Zealand, ${ }^{3}$ Howard Hughes Medical Institute and Department of Brain and Cognitive Sciences, Massachusetts Institute of Technology, \\ Cambridge, Massachusetts 02139, and ${ }^{4}$ The Salk Institute for Biological Studies, La Jolla, California 92037
}

Understanding how neural circuits work requires a detailed knowledge of cellular-level connectivity. Our current understanding of neural circuitry is limited by the constraints of existing tools for transsynaptic tracing. Some of the most intractable problems are a lack of cellular specificity of uptake, transport across multiple synaptic steps conflating direct and indirect inputs, and poor labeling of minor inputs. We used a novel combination of transgenic mouse technology and a recently developed tracing system based on rabies virus (Wickersham et al., 2007a,b) to overcome all three constraints. Because the virus requires transgene expression for both initial infection and subsequent retrograde transsynaptic infection, we created several lines of mice that express these genes in defined cell types using the tetracycline-dependent transactivator system (Mansuy and Bujard, 2000). Fluorescent labeling from viral replication is thereby restricted to defined neuronal cell types and their direct monosynaptic inputs. Because viral replication does not depend on transgene expression, it provides robust amplification of signal in presynaptic neurons regardless of input strength. We injected virus into transgenic crosses expressing the viral transgenes in specific cell types of the hippocampus formation to demonstrate cell-specific infection and monosynaptic retrograde transport of virus, which strongly labels even minor inputs. Such neuron-specific transgenic complementation of recombinant rabies virus holds great promise for obtaining cellular-resolution wiring diagrams of the mammalian CNS.

\section{Introduction}

The mammalian CNS is composed of an immensely large number of neurons, each typically connected to thousands of other neurons. A key step toward developing a functional understanding of a neural circuit is elucidating its connectivity, which is best achieved by transsynaptic tracers. Neuroanatomists have used a wide variety of both anterograde and retrograde tracers ranging from lectins (Köbbert et al., 2000) to viruses (Kelly and Strick, 2000; Ugolini, 2008) to obtain a general understanding of the connectivity of the brain. However, there are limitations to prior approaches that preclude a detailed understanding of the brain's circuitry at the cellular level (Luo et al., 2008). Key limitations include a lack of cellular specificity of uptake of label, difficulties in determining whether a given neuron is monosynaptically or polysynaptically connected to the initially labeled neurons, and highly variable intensity of labeling making it especially difficult to visualize minor inputs. We present an approach combining a

\footnotetext{
Received May 11, 2010; revised Aug. 30, 2010; accepted 0ct. 14, 2010.

Supported by National Institute of Mental Health Grant R21 MH076289 and Department of Defense Grant W81XWH-09-2-0114 to C.G.K. and National Institute of Neurological Disorders and Stroke Grant RC2 NS069464 to E.M.C. and C.G.K., and Howard Hughes Medical Institute to H.S.S. and I.R.W. We thank Heather Sullivan of the Seung Lab for outstanding technical assistance and Dr. Karl-Klaus Conzelmann for starting stocks of SADdG-mCherry.

${ }^{*}$ A.P.W. and L.S. contributed equally to this manuscript.

Correspondence should be addressed to Clifford G. Kentros, 1254 University of Oregon, Eugene, 0R 97403. E-mail: cliff@uoneuro.uoregon.edu.

DOI:10.1523/JNEUROSCI.2442-10.2010

Copyright $\odot 2010$ the authors $\quad$ 0270-6474/10/3016509-05\$15.00/0
}

recombinant rabies virus with novel transgenic mice that, for the first time, overcomes all three of these constraints, enabling the unequivocal determination of direct monosynaptic inputs of defined neuronal cell types.

Wild-type rabies virus infects neurons nonspecifically and spreads retrogradely across synapses. The recombinant virus has the gene of the rabies virus envelope glycoprotein (RG), responsible for infectivity and transsynaptic spread, deleted from its genome and replaced with that of the fluorophore mCherry (see Materials and Methods). The rabies virus used for injections [SAD $\Delta \mathrm{G}-\mathrm{mCherry}(\mathrm{EnvA})]$ has (i.e., is pseudotyped with) a fusion protein between the cytoplasmic domain of RG and the avian sarcoma and leukosis virus protein EnvA in its viral envelope so it can only infect cells that express EnvA's receptor, TVA (Wickersham et al., 2007a). Since mammalian neurons do not express TVA, the injected virus cannot infect wild-type neurons. After infecting a TVA-positive neuron, the virus can replicate, strongly labeling first-order (i.e., initially infected) neurons, but since its genome lacks RG, it cannot infect other neurons by itself. Thus, infection is specified by transgene expression, retrograde transport is limited to a single synapse, and unfettered viral replication provides powerful amplification of signal.

We therefore combined the transsynaptic specificity and signal amplification afforded by this viral system with the anatomical specificity of mouse molecular genetics to enable the determination of the monosynaptic inputs to specific neuronal cell types in intact brains. We generated eight lines of transgenic 
mice capable of expressing both transgenes (TVAG) from a tetracycline-responsive hybrid promoter $\left(\mathrm{pTRE}_{\text {tight }}\right)$ (supplemental Fig. S1, available at www.jneurosci.org as supplemental material) when crossed to transactivator lines expressing either a tetracycline-dependent transactivator (tTA; tet-off) or reverse tTA (tet-on) fusion protein (for background, see Mansuy and Bujard, 2000). These lines were then crossed to several neuronspecific tTA lines we created using the CaMKII $\alpha$ promoter, and were used to demonstrate neuron-specific monosynaptic connectivity via stereotactic injections of recombinant virus into the hippocampal formation of double-positive (tTA and TVAG, or $+/+)$ animals.

\section{Materials and Methods}

Generation of biological reagents. EnvA-pseudotyped lentivirus was produced as previously described (Wickersham et al., 2007a) but with the VSV glycoprotein expression vector replaced with the plasmid pCMMPEnvARGCD-IRES-EGFP (Wickersham et al., 2007b) encoding the EnvA envelope glycoprotein with the cytoplasmic domain of the rabies virus glycoprotein. Starting stocks of rabies virus with the mCherry gene substituted for that of the viral glycoprotein were a generous gift from Dr. Karl-Klaus Conzelmann, and EnvA-pseudotyped concentrated stocks were produced and pseudotyped with the EnvA envelope protein (Wickersham et al., 2007b). A 2441 bp fragment containing a myc-tagged EnvA-receptor (TVA) and the rabies viral coat protein (RG), separated by the foot and mouth disease virus 2A element (Szymczak et al., 2004), was excised from the parent plasmid and cloned directionally into the multiple cloning site of the $\mathrm{pTRE}_{\text {tight }}(\mathrm{pTT})$ plasmid (Clontech), which contains a tetracycline-transactivator-dependent promoter designed specifically for tight transcriptional control. The resulting construct was cut and gel-purified for pronuclear injection. For the transactivator lines, a plasmid containing the calcium/calmodulin-dependent kinase II $\alpha$ subunit (CK2) promoter driving a codon-optimized tetracycline transactivator (tTA, or tet-off) was flanked with loxP sites to allow excision with Cre recombinase. The resulting construct was cut to remove the vector arms and gel-purified for pronuclear injection. We also used a commercially available transactivator line that expresses tTA in the forebrain from the CaMKII $\alpha$ promoter [B6; CBA-Tg(Camk2atTA) 1Mmay/J mouse; Jackson Laboratories, strain \# 003010)], which typically led to expression levels that were too dense for our purposes.

Histological and anatomical procedures. All procedures were performed in accordance with guidelines approved by University of Oregon's Animal Care and Use Committee and the National Institutes of Health Guide for the Care and Use of Laboratory Animals (NIH Publications No. 80-23). A total of 46 mice were injected with virus in the present study (supplemental Table S1, available at www.jneurosci.org as supplemental material). Genotyping was performed via PCR with two sets of primers (supplemental Fig. S1, available at www.jneurosci.org as supplemental material) for each transgenic line in a cross. Injections into area CA1 of the hippocampus ( $50 \mathrm{nl}$ of rabies virus; titer, $9.9 \times 10^{8} / \mathrm{ml}$ ) were made at anteroposterior (AP) $-2.0 \mathrm{~mm}$ and mediolateral (ML) $1.8 \mathrm{~mm}$ from bregma at a depth (dorsoventral, DV) of $1.1 \mathrm{~mm}$. Injections into the subicular complex (150 $\mathrm{nl}$ rabies virus; $150 \mathrm{nl}$ lentivirus; titer, $9.4 \times$ $10 \% / \mathrm{ml}$ ) were made at AP $-3.0 \mathrm{~mm}$ and ML $1.8 \mathrm{~mm}$ from bregma at a depth of $1.7 \mathrm{~mm}$. Additional control injections were also made into M1 (50 $\mathrm{nl}$ rabies virus; AP, $1.0 \mathrm{~mm}$; ML, $1.3 \mathrm{~mm}$; DV, $0.6 \mathrm{~mm}$ ), entorhinal cortex ( $50 \mathrm{nl}$ rabies virus; $\mathrm{AP},-4.8 \mathrm{~mm}$; ML, $3.1 \mathrm{~mm}$; DV, $1.0 \mathrm{~mm}$ ), and the amygdala (100 $\mathrm{nl}$ rabies virus; $\mathrm{AP},-1.0 \mathrm{~mm}$; ML, $3.0 \mathrm{~mm}$; DV, 4.0 $\mathrm{mm}$ ). The true wild-type (TVA-negative) animals had no way of expressing TVA, whereas the TRE-leak controls had no tTA, so controlled for the leak of the TRE lines. The tTA leak controls were of two types: injections into regions known not to express TVA mRNA, and injections into animals that were genotypically double-positive but nonetheless failed to express detectable amounts of TVA mRNA in the target structure (although most did express TVA mRNA in a few neurons in other structures, confirming the genotyping results).

Mice were killed 7 (rabies virus) or 16 (lentivirus) d following surgery; perfusions and tissue sectioning ( $30 \mu \mathrm{m}$ thick) were performed as described previously (Wehr et al., 2009). All sections were kept at room temperature for $24 \mathrm{~h}$, then either processed with in situ hybridization (ISH) for TVA mRNA expression and fluorescent Nissl staining or stored at $-80^{\circ} \mathrm{C}$.

Sections were viewed using a Zeiss Axioplan microscope. All photomicrographs of virus-labeled tissue were captured before processing for mRNA expression or Nissl fluorescence. Photomicrographs were taken with a Nikon Coolpix 3.34MPixel camera. No image postprocessing was performed on photomicrographs illustrating infection or transport. In some instances, contrast and brightness were adjusted to enhance Nissl or mRNA image clarity, but always to the same levels in any single composite image or figure. All mCherry fluorescing neurons within the boundaries of a given illustration are shown. Composites and photooverlays were created using Canvas X software (ACD Systems).

Nonradioactive ISH was performed as described previously (Wehr et al., 2009). The riboprobe template was cloned from a fragment of the TVA gene excised from the pTT/TVAG construct and ligated into pBSKS+ (supplemental Fig. S1, available at www.jneurosci.org as supplemental material). A 492 bp transcript was generated using the BamHI linearized pTVA riboprobe template in the presence of T3 RNA polymerase and dig-labeled nucleotides. Following overnight incubation at room temperature, slices were washed at room temperature with MABT buffer $5 \times 5^{\prime}$, and then alkaline phosphatase-staining buffer $2 \times 10^{\prime}$, after which $3.5 \mu \mathrm{l} / \mathrm{ml} \mathrm{NBT}, 2.6 \mu \mathrm{l} / \mathrm{ml}$ BCIP, and $80 \mu \mathrm{l} / \mathrm{ml}$ levamisole were added and the colorimetric reaction was allowed to develop for $3 \mathrm{~h}$ at $37^{\circ} \mathrm{C}$ under agitation. The reaction was stopped by two washes with PBS $(0.1 \%$ Tween 20$)$, then two washes in deionized $\mathrm{H}_{2} \mathrm{O}$. Following ISH, sections underwent processing for a fluorescent Nissl stain (a 1:30 PBS dilution of NeuroTrace; N21480 or N21482; Invitrogen).

Tissue was examined (blind to genotype) immediately following sectioning to identify the location of the injection site and photomicrograph and catalog any fluorescent signal associated with viral infection and/or transport. Following processing for mRNA and Nissl staining, photomicrographs were taken of those sections in which infection and/or transport had been evident, and the images were superimposed for analysis.

\section{Results}

\section{Specificity of infection}

To demonstrate cell-specific infection, the TRE-TVAG lines were crossed to several tTA driver lines we created with a range of forebrain-specific tTA expression based upon the CaMKII $\alpha$ promoter (Mayford et al., 1996) and injected with virus (supplemental Table S1, available at www.jneurosci.org as supplemental material). Each cross had distinct transgene expression patterns, ranging from extremely sparse in a single neuronal subtype to a relatively dense expression across most primary neurons of the forebrain. Although the more densely expressing crosses provide the most aesthetically striking images (e.g., Fig. $3 D, E$ ), those that express very sparsely are arguably more informative since one can more easily count infected neurons and visualize individual processes. The most straightforward demonstration of cell-specific infection would be double-labeling with mCherry and TVA mRNA, but this was complicated by the fact that rabies viral infection appears to disrupt transgene expression. We saw an inverse relationship between mRNA and mCherry labeling density (supplemental Fig. S2, available at www.jneurosci.org as supplemental material), presumably due to viral infection. We circumvented this issue by injecting a GFP-labeled lentivirus [LV-CMVP-eGFP(EnvA)] pseudotyped with the same EnvA-RG fusion protein as the injected rabies virus to demonstrate specificity of infection. Since the lentivirus is incapable of transsynaptic transport, labeled neurons must be the result of direct infection. This demonstrates the restriction of infection to TVA-positive neurons; every one of the minority of neurons infected with the lentivirus were found to also express TVA mRNA (Fig. 1). 


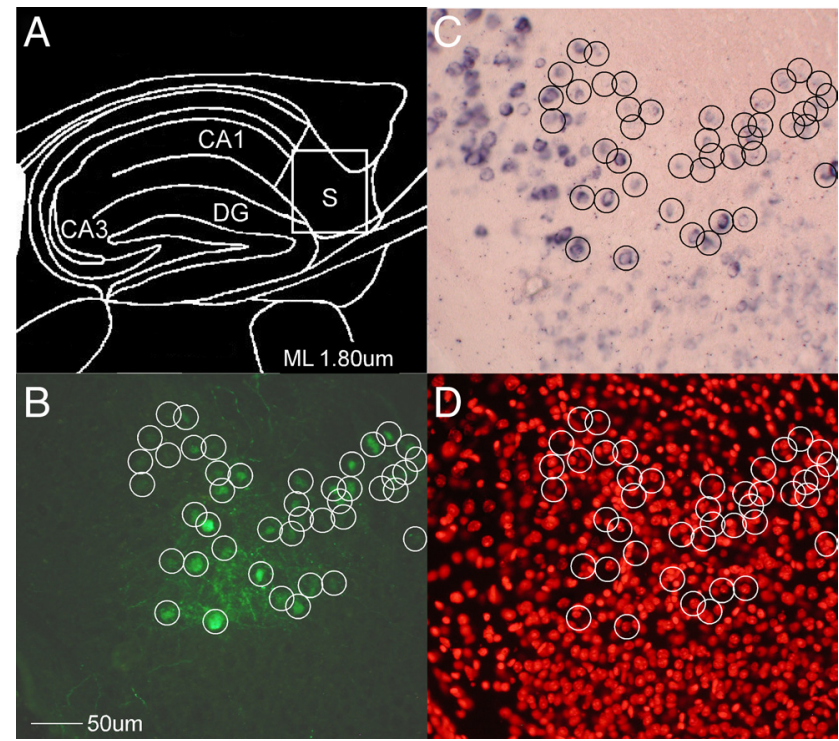

Figure 1. Transgenic specification of viral infection. $A$, Location of injection site of EnvApseudotyped lentivirus control. $\boldsymbol{B}-\boldsymbol{D}$, All infected (green) neurons were encircled and compared with TVA ISH $(\boldsymbol{B})$ to determine transgene expression $(\boldsymbol{C})$ and a Nissl stain to count nuclei $(\boldsymbol{D})$. Although only a minority of neurons were mRNA-positive (191/1015), every infected neuron was mRNA-positive (43/43), suggesting restriction of infection to transgenic neurons. CA1, Field CA1 of hippocampus; $C A 3$, field CA3 of hippocampus; $D G$, dentate gyrus; $S$, subiculum.

Since the recombinant virus is replication-competent, infection of a single virion is theoretically all that is necessary for label. The resulting unambiguous label of even minor inputs is one of the positive features of this approach, but it has a potential downside: it is possible that leaky expression of extremely small amounts of TVA could result in labeled neurons around the injection site even without detectable levels of mRNA. Since this would undermine the specificity at the core of this approach, we took considerable pains to investigate the tightness of infection by performing three distinct kinds of control injections (see supplemental Table S1, available at www.jneurosci.org as supplemental material, for a summary of all injections). Wild-type injections were into animals genetically incapable of expressing any viral proteins (i.e., TVAG -/tTA - and TVAG -/tTA + littermates), providing a measure of the recombinant virus' ability to infect tissue. However, leaky expression from either of the two transgenic lines of a cross (tTA $\times$ TVAG) might also lead to infection, so we also performed two kinds of control injections in TVAG + animals. One kind (TRE-leak controls) involved injecting virus into TVAG +/tTA - animals to test for tTAindependent transcription from the hybrid TRE promoter. Finally, just as the hybrid promoter driving the TVA can leak, so can the promoter which drives the tTA, which might similarly decrease specificity by driving tTA-dependent TVA expression outside of the cells of interest. We tested for this potential tTA-leak by injecting virus into brain areas that did not exhibit TVA mRNA in TVAG $+/ \mathrm{tTA}+$ animals, both by choosing areas that did not express mRNA and because the sparsest crosses (e.g., those involving line TVAG2) sometimes did not express appreciable amounts of TVA mRNA.

In all cases, robust uptake, replication, and retrograde transport of the recombinant rabies virus were observed exclusively in $+/+$ mice (supplemental Table S1, available at www.jneurosci. org as supplemental material). The vast majority of wild-type control injections (13/14 TVAG-negative animals) resulted in absolutely no infection, but consistent with the known low-level

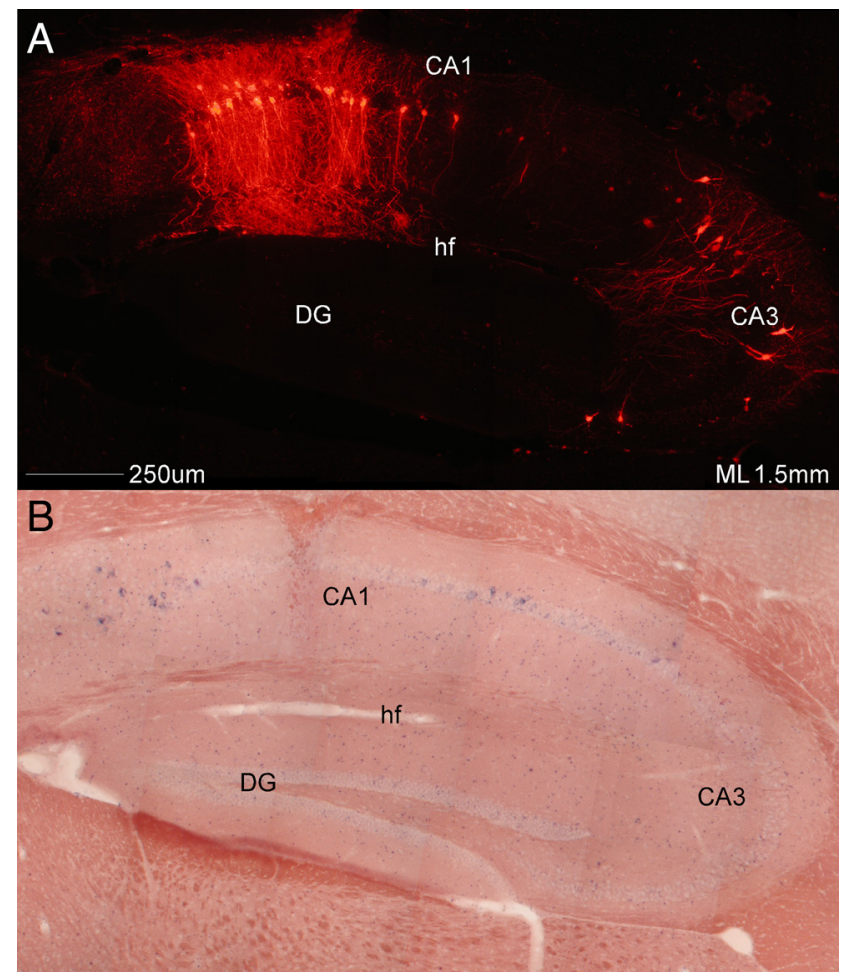

Figure 2. Monosynaptic restriction of viral transport. A sagittal section from a transgenic cross injected with $S A D \Delta G-m C h e r r y(E n v A)$ in the CA1 pyramidal cell layer. $A$, Viral mCherry label alone. $\boldsymbol{B}$, Same section as $\boldsymbol{A}$ hybridized to TVA mRNA. This cross only expresses TVA mRNA in a small minority of neurons in the subiculum and CA1 pyramidal cell layer, not in CA3 pyramidal neurons. As can be seen in $A$, viral transport is restricted to direct presynaptic inputs of $C A 1$ pyramidal neurons (CA3 neurons), with no label in dentate granule neurons, known to be presynaptic to CA3 pyramidal neurons. Note the anticorrelation between mRNA expression and viral signal around the injection site, presumably caused by viral interference with host cell gene expression. CA1, Field CA1 of hippocampus; CA3, field CA3 of hippocampus; DG, dentate gyrus; hf, hippocampal fissure.

tropism of EnvA for mammalian cells (Wickersham et al., 2007a,b), one injection into a wild-type animal resulted in three labeled neurons near the injection site. Three TRE-leak (tTA-I TRE-TVAG + ) controls also exhibited infection of a few neurons $(7 \pm 4)$. However, since they were all from the same line (TVAG6) and none of the other TRE-leak controls exhibited any infection, we concluded that this leakiness was specific to the insertion site of this line, which we have since terminated. Perhaps the most convincing demonstration of the specificity of infection comes from the tTA-leak controls, i.e., genotypically $+/+$ animals, which exhibited no TVA mRNA around the injection site. Of nine such injections, seven yielded no infected cells, whereas the remaining two averaged four fluorescent neurons. It is worth noting that these latter two animals were of a cross that in other $+/+$ animals exhibited sparse but detectable mRNA expression in CA1.

Although a slight minority of each of these three kinds of control injections resulted in infection of a handful of neurons, none that did so exhibited viral label $>200 \mu \mathrm{m}$ away from the injection site, nor in any neurons known to be presynaptic to the infected neurons. This suggests that, although occasionally strain-specific leak of transgene mRNA may trigger infection in mRNA-negative neurons, such neurons do not express sufficient viral glycoprotein to support the assembly of infectioncompetent rabies virus particles. Thus, infection appears to be tightly controlled by transgene expression, with all but one of the 
TVAG strains exhibiting EnvAmediated infection only when the injection targets neurons clearly expressing TVA mRNA. Furthermore, the retrograde transport of label is exclusive to mRNA-positive neurons.

\section{Monosynaptic transport}

We chose to concentrate our efforts on CA1 pyramidal neurons since not only is their connectivity extremely well characterized, they are one of the few neuronal cell types known to have vanishingly few interconnections (for review, see Amaral and Lavenex, 2007), which greatly facilitates the identification of first-order neurons. We therefore used crosses that sparsely express the viral transgenes in a small minority of CA1 pyramidal neurons to provide some degree of quantification of transsynaptic viral label and to demonstrate the restriction of retrograde transport to a single synapse. Following injection of virus into dorsal CA1 stratum pyramidale, mCherry label was observed around the injection site in a small subset of pyramidal neurons and putative interneurons (Fig. 2 and supplemental Fig. S2, available at www.jneurosci.org as supplemental material). As noted above, the viral label and TVA mRNA are of a similar density in CA1 and are anticorrelated. Together, they closely resemble the pattern seen with TVA mRNA in the contralateral hippocampus, suggesting direct infection only of transgenic CA1 pyramidal neurons (supplemental Fig. S2, available at www.jneurosci.org as supplemental material).

The present results demonstrate convincingly that transsynaptic label is restricted to a single synapse. This is most obviously seen by the complete absence of label in dentate gyrus (DG) (Fig. 2, supplemental Table S2, available at www. jneurosci.org as supplemental material), which projects to CA3 but not CA1 (for review, see Amaral and Lavenex, 2007). Since it has been estimated that rabies virus requires only $24 \mathrm{~h}$ to traverse a synapse (Ugolini, 2008), it is very unlikely that this was due to inadequate postinfection time ( $7 \mathrm{~d})$. However, even when only a handful of CA1 pyramidal neurons were infected, robust label of individual neurons was seen in their major monosynaptic inputs, such as ipsilateral and contralateral CA3 (Fig. 2 and supplemental Fig. S3, available at www.jneurosci.org as supplemental material) and the entorhinal cortex (supplemental Fig. S3, available at www.jneurosci.org as supplemental material) (for review, see Amaral and Lavenex, 2007).

\section{Unambiguous label of minor monosynaptic inputs}

Because the virus is intracellularly replication-competent, infection by even a single viral particle should result in strong label of a neuron (Wickersham et al., 2007a). Although this fact makes this approach very susceptible to leak, as discussed above, it does solve another perennial problem in neuroanatomy by strongly labeling neurons of relatively minor inputs. Figure 3 demonstrates strong retrograde labeling of neurons in areas known to directly project more weakly to hippocampal pyramidal neurons (for review, see Amaral and Lavenex, 2007), such as limbic thalamus (Fig. $3 A, C$ ), ventral tegmental area (Fig. $3 B$ ), and the basal forebrain cholinergic nuclei (Fig. $3 D, E$ ), as well as in presumed CA1 interneurons and neurons of the locus ceruleus (data not shown). These data illustrate the ability of viral replication to amplify the signal from weak inputs, labeling these neurons as clearly as those providing major synaptic inputs.

\section{Discussion}

The above data illustrate the great potential of this combined viral and transgenic approach. Cellular specificity of tracer uptake has been achieved before by both viral (DeFalco et al., 2001) and transgenic (Braz et al., 2002) means, but no prior approach also has the ability to both restrict transport to a single synaptic step and obtain robust signal regardless of input strength. Thus, this 
combination of transgenic mice designed to complement recombinant rabies virus appears to solve some of the most vexing problems facing investigators trying to determine the connectivity of the mammalian CNS. The viral approach has been described before. The transgenes necessary for infection and transport were randomly introduced into cultured brain slices (Wickersham et al., 2007a,b) or into a single cortical cell (Marshel et al., 2010). However, our pyramidal cell data most unambiguously demonstrate the system's most striking feature, the restriction of transport to a single synapse. Moreover, the transgenic approach provides valuable modularity to the system; these TRETVAG lines can be crossed to any neuron-specific tetracycline transactivator line to confer its specificity of infection, potentially allowing investigation of the connectivity of any neuronal cell type for which a tTA driver line exists. Thus, the distribution of these lines to the scientific community should enable the determination of the direct monosynaptic inputs to potentially any neuronal cell type of interest.

Although the above properties make this a uniquely powerful approach, it is not without its limitations. The relatively small proportion of labeled presynaptic CA3 pyramidal neurons is perplexing. Prior estimates suggest that a single CA3 pyramidal neuron may synapse onto as many as 30,000 to 60,000 CA1 pyramidal neurons (Li et al., 1994). As illustrated in supplemental Figure S4 (available at www.jneurosci.org as supplemental material), the ratio of CA3 (second-order) to CA1 pyramidal neurons we observed in the four CA1 infections we quantified is consistently many orders of magnitude lower (supplemental Table S2, available at www.jneurosci.org as supplemental material). Transformation-induced downregulation of transgenic glycoprotein expression may limit the amount of infectious virus being made in first-order neurons, which would lead to viral labeling in only a small subset of upstream neurons. There may be a critical concentration of the viral glycoprotein necessary for assembly of infection-competent virions, so the level of transgene expression at time of infection may dictate the proportion of upstream neurons labeled. Also, although neurons looked cytoarchitecturally normal, the downregulation of host cell mRNA expression (supplemental Fig. S2, available at www.jneurosci.org as supplemental material) caused by viral infection may have deleterious effects upon infected neurons, though this is more significant issue for using rabies virus as a vector than as a tracer.

Nevertheless, even if the system cannot give the precise number of inputs to a neuron, this drawback is shared with all competing approaches. For instance, the counting of varicosities of filled neurons used to estimate CA3-CA1 connectivity is fraught with its own caveats and is particularly prone to overestimates of functional synapses. Moreover, the system may still provide useful quantitative data if it is off by a consistent scalar, as the similarity in CA1-CA3 ratios for the four injections we quantified suggests (supplemental Fig. S4 and Table S2, available at www. jneurosci.org as supplemental material).
All in all, the combined features of cellular specificity, monosynaptic transport, and unambiguous label make this approach uniquely valuable; labeling of the monosynaptic connections of defined cell types is of significant value. However, the specificity of the system will demand a commensurate amount of effort to determine the cell types of the first- and second-order neurons; the cellular selectivity is wasted unless the identities of the labeled neurons are determined with equal granularity. Provided these challenges are met, the transgenic complementation of recombinant rabies virus presented here will be an invaluable tool for systems neuroscience, enabling the determination of the connectivity of the mammalian brain with the cellular-level resolution necessary for accurate circuit modeling and reconstruction (Seung, 2009).

\section{References}

Amaral D, Lavenex P (2007) The hippocampus book, pp 37-114. Oxford, NY: Oxford UP.

Braz JM, Rico B, Basbaum AI (2002) Transneuronal tracing of diverse CNS circuits by Cre-mediated induction of wheat germ agglutinin in transgenic mice. Proc Natl Acad Sci U S A 99:15148-15153.

DeFalco J, Tomishima M, Liu H, Zhao C, Cai X, Marth JD, Enquist L, Friedman JM (2001) Virus-assisted mapping of neural inputs to a feeding center in the hypothalamus. Science 291:2608-2613.

Kelly RM, Strick PL (2000) Rabies as a transneuronal tracer of circuits in the central nervous system. J Neurosci Methods 103:63-71.

Köbbert C, Apps R, Bechmann I, Lanciego JL, Mey J, Thanos S (2000) Current concepts in neuroanatomical tracing. Prog Neurobiol 62:327-351.

Li XG, Somogyi P, Ylinen A, Buzsáki G (1994) The hippocampal CA3 network: an in vivo intracellular labeling study. J Comp Neurol 339:181-208.

Luo L, Callaway EM, Svoboda K (2008) Genetic dissection of neural circuits. Neuron 57:634-660.

Mansuy IM, Bujard H (2000) Tetracycline-regulated gene expression in the brain. Curr Opin Neurobiol 10:593-596.

Marshel JH, Mori T, Nielsen KJ, Callaway EM (2010) Targeting single neuronal networks for gene expression and cell labeling in vivo. Neuron 67:562-574.

Mayford M, Bach ME, Huang YY, Wang L, Hawkins RD, Kandel ER (1996) Control of memory formation through regulated expression of a CaMKII transgene. Science 274:1678-1683.

Seung HS (2009) Reading the book of memory: sparse sampling versus dense mapping of connectomes. Neuron 62:17-29.

Szymczak AL, Workman CJ, Wang Y, Vignali KM, Dilioglou S, Vanin EF, Vignali DA (2004) Correction of multi-gene deficiency in vivo using a single 'self-cleaving' $2 \mathrm{~A}$ peptide-based retroviral vector. Nat Biotechnol 22:589-594.

Ugolini G (2008) Use of rabies virus as a transneuronal tracer of neuronal connections: implications for the understanding of rabies pathogenesis. Dev Biol (Basel) 131:493-506.

Wehr M, Hostick U, Kyweriga M, Tan A, Weible AP, Wu H, Wu W, Callaway EM, Kentros C (2009) Transgenic silencing of neurons in the mammalian brain by expression of the allatostatin receptor (AlstR). J Neurophysiol 102:2554-2562.

Wickersham IR, Finke S, Conzelmann KK, Callaway EM (2007a) Retrograde neuronal tracing with a deletion-mutant rabies virus. Nat Methods 4:47-49.

Wickersham IR, Lyon DC, Barnard RJ, Mori T, Finke S, Conzelmann KK, Young JA, Callaway EM (2007b) Monosynaptic restriction of transsynaptic tracing from single, genetically targeted neurons. Neuron 53:639647. 